\title{
Control and stability on chaotic convection in porous media with time delayed fractional orders
}

\section{Khaled Moaddy*}

"Correspondence: moaddy@su.edu.sa Department of Mathematics, Faculty of Science and Arts, Shaqra University, Shaqra, 11691, Saudi Arabia

\section{Springer}

\begin{abstract}
In this paper, we study the effect of time delay and the scaled Rayleigh number on chaotic convection in porous media with fractional order. The stability analysis for different fractional-order cases is investigated and the effective chaotic range of the fractional order is determined by a general synchronization of nonidentical chaotic systems based on the active control technique. The numerical results demonstrate that the effect of various values of the scaled Rayleigh number $R$, time delay and fractional orders changes the chaotic convection behavior to limit cycles or stable system in porous media.
\end{abstract}

Keywords: chaotic behavior; fractional differential equations; synchronization; internal heat generation; porous media

\section{Introduction}

There is a great need to control or obtain accurate numerical results for chaotic convection as chaos theory plays an important role in industrial applications, particularly in chemical reactions, biological systems [1], information processing, secure communications [2], electronics [3, 4], and with memristors [5]. Recently, control and synchronization of fractional chaotic systems has raised up some problems [6-8]. The consistency of the improvement of models based on fractional order differential structure has had increased reputation in the research of dynamical systems [9-11]. Chaos synchronization plays an important role in secure communication, digital cryptography, image encryption, signal and control processing [12-19]. Much attention has been devoted to the search for better and more efficient methods for the control or determination of a solution, approximate or analytical, of chaotic systems $[9,10,13,20,21]$. Yu et al. studied the synchronization of three chaotic fractional-order Lorenz systems with bidirectional coupling [20]; Odibat et al. [21] investigated the chaos synchronization of two identical systems via linear control; Bhalekar and Daftardar-Gejji [22] demonstrated that two different fractional-order chaotic systems can be synchronized using active control. Zhen et al. [23] implemented the delayed fractional-order chaotic logistic system in a simple chaotic masking method to illustrate the security enhancement. Daftardaret et al. investigated the effect of delay on chaos in the fractional-order Chen system [24].

(c) The Author(s) 2017. This article is distributed under the terms of the Creative Commons Attribution 4.0 International License (http://creativecommons.org/licenses/by/4.0/), which permits unrestricted use, distribution, and reproduction in any medium, provided you give appropriate credit to the original author(s) and the source, provide a link to the Creative Commons license, and indicate if changes were made. 
More recently, chaotic behavior in a fluid-saturated porous medium has attracted interest due to its wide application in such fields as geothermal energy utilization, oil reservoir modeling, catalytic packed beds filtration, thermal insulation and nuclear waste disposal [25]. It has been observed in many natural systems, such as the time evolution of the magnetic field of celestial bodies, molecular vibrations, the dynamics of satellite in the solar system, the weather, in ecology and in neurons [26, 27]. Moaddy et al. [28] studied the effect of the fractional-order chaotic behavior of nanofluids in a fluid layer heated from below; Jawdat et al. [29] investigated the effects of a uniform internal heat generation on chaotic behavior in thermal convection in a fluid-saturated porous layer subject to gravity and heated from below for low Prandtl number.

The stability analysis of fractional differential equations is very important according to the required application behavior. Each behavior is related to the location of the system's poles with respect to the equilibrium points. In this paper we will study the eigenvalue problem for the fractional-order case for different values of Rayleigh number $\mathrm{R}$, while the stability of integer-order case was studied in [29].

Motivated by the above discussions, in this paper we have four aims, where the first aim is to extend the work of Jawdat et al. [29] to study the effect of the fractional order on chaotic behavior in a fluid-saturated porous layer subject to gravity and heated from below for low Prandtl number. The second aim is to study the property of time delay with fractional-order range which exhibits chaotic behavior for the chaotic system of fractional orders. Several cases are investigated for different fractional orders changing only a single system parameter. Stable, periodic and chaotic responses are shown for each system parameter but with different fractional-order ranges. The third aim is to discuss the stability analysis of the fractional-order system for different order and different values of Rayleigh number $R$. The last aim is to investigate the synchronization of different fractional-order chaotic systems; the numerical solutions of the master, slave and error systems using Adams-Bashforth-Moulton predictor corrector algorithm are proposed.

\section{The proposed system}

In this section we study the effect of time delay and fractional order on chaotic convection in porous media. Jawdat et al. [29] provided the following system of differential equations:

$$
\begin{aligned}
& \dot{X}=\delta(Y-X), \\
& \dot{Y}=\hat{R} X-(1-b) Y-(\hat{R}-1+b) X Z, \\
& \dot{Z}=(4 \gamma-b)(X Y-Z) .
\end{aligned}
$$

Eqs. (1)-(3) are equivalent to the Lorenz equations [30, 31] although with different coefficients. For porous media convection, the value of $\gamma=0.5$ [26]. With this value of $\gamma$, the definitions of $R, \hat{R}, \delta$ and $b$ are explicitly expressed in the forms [29] $R=R_{a} / 4 \pi^{2}$, $\hat{R}=R_{a} /\left(4 \pi^{2}-G\right), \delta=V_{a} / 2 \pi^{2}$, and $b=G / 2 \pi^{2}$. When $b=0$, system (1)-(3) reduces to that of Vadasz and Olek [26].

In this work we consider the generalization of system (1)-(3) which involves fractional order with time delay

$$
D^{\alpha} X(t)=\delta(Y(t)-X(t-\tau)),
$$




$$
\begin{aligned}
& D^{\alpha} Y(t)=\hat{R} X(t-\tau)-Y(t)-(\hat{R}-1) X(t-\tau) Z(t), \\
& D^{\alpha} Z(t)=(4 \gamma-b)(X(t-\tau) Y(t)-Z(t)),
\end{aligned}
$$

where $X(t)=0.2, Y(t)=0$ and $Z(t)=0.5$ for $t \in[-\tau, 0]$.

Applying the Adams-Bashforth-Moulton predictor-corrector algorithm is proposed by Diethelm et al. [32, 33] to solve the fractional-order differential equations, which yields

$$
\begin{aligned}
X_{n+1}(t)= & X_{0}(t)+\frac{h^{\alpha}}{\Gamma(\alpha+1)}\left(\sum_{j=0}^{n} \alpha_{1, j, n+1}\left[\delta\left(Y_{j}(t)-X_{j}(t)\right)\right]\right. \\
& \left.+\left[\delta\left(Y_{n+1}^{p}(t)-X_{n+1}^{p}(t)\right)\right]\right), \\
Y_{n+1}(t)= & Y_{0}(t)+\frac{h^{\alpha}}{\Gamma(\alpha+2)}\left(\sum _ { j = 0 } ^ { n } \alpha _ { 2 , j , n + 1 } \left[\hat{R} X_{j}(t-\tau)\right.\right. \\
& \left.-(1-b) Y_{j}(t)-(\hat{R}-1+b) X_{j}(t-\tau) Z_{j}(t)\right] \\
& +\left[\hat{R} X_{n+1}^{p}(t-\tau)-(1-b) Y_{n+1}^{p}(t)\right. \\
& \left.\left.-(\hat{R}-1+b) X_{n+1}^{p}(t-\tau) Z_{n+1}^{p}(t)\right]\right), \\
Z_{n+1}(t)= & Z_{0}(t)+\frac{h^{\alpha}}{\Gamma(\alpha+2)}\left(\sum_{j=0}^{n} \alpha_{3, j, n+1}\left[(4 \gamma-b)\left(X_{j}(t-\tau) Y_{j}(t)-Z_{j}(t)\right)\right]\right. \\
& \left.+\left[(4 \gamma-b)\left(X_{n+1}^{p}(t-\tau) Y_{n+1}^{p}(t)-Z_{n+1}^{p}(t)\right)\right]\right),
\end{aligned}
$$

where

$$
a_{j, n+1}= \begin{cases}n^{\alpha+1}-(n-\alpha)(n+1)^{\alpha}, & j=0, \\ (n-j+2)^{\alpha+1}+(n-j)^{\alpha+1}-2(n-j+1)^{\alpha+1}, & 1 \leq j \leq n, \\ 1, & j=n+1 .\end{cases}
$$

$\left\{t_{n}=n h, n:-k,-k+1, \ldots,-1,0,1, \ldots, N\right\}, n$ and $k$ are integers, such that $h=T / N, T=\tau / k$, and

$$
\begin{aligned}
X_{n+1}^{p}(t)= & X_{0}(t)+\frac{1}{\Gamma(\alpha)}\left(\sum_{j=0}^{n} b_{1, j, n+1}\left[\delta\left(Y_{j}(t)-X_{j}(t-\tau)\right)\right]\right), \\
Y_{n+1}^{p}(t)= & Y_{0}(t)+\frac{1}{\Gamma(\alpha)}\left(\sum _ { j = 0 } ^ { n } b _ { 2 , j , n + 1 } \left[\hat{R} X_{j}(t)-(1-b) Y_{j}(t)\right.\right. \\
& \left.\left.-(\hat{R}-1+b) X_{j}(t-\tau) Z_{j}(t)\right]\right), \\
Z_{n+1}^{p}(t)= & Z_{0}(t)+\frac{1}{\Gamma(\alpha)}\left(\sum_{j=0}^{n} b_{3, j, n+1}\left[(4 \gamma-b)\left(X_{j}(t-\tau) Y_{j}(t)-Z_{j}(t)\right)\right]\right),
\end{aligned}
$$


where $b_{j, n+1}=\frac{h^{\alpha}}{\alpha}(n-j+1)^{\alpha}-(n-j)^{\alpha}$ and $0 \leq j \leq n$. The estimated error of this method is $e=\max \left|X\left(t_{j}\right)-X_{h}\left(t_{j}\right)\right|=\phi\left(h^{\rho}\right)$, where $\rho=\min (2,1+\alpha), j=1,2, \ldots, N$.

\section{Stability analysis}

One of the methods to study the stability of fractional-order systems is the $W$-plane method [15] which can be used for different fractional orders inside the same system. There are two planes in the fractional-order case where the first is the so-called $W$-plane which has all poles either in physical or nonphysical planes. The second is the $s$-plane where the physical poles for different values of $R$ in the $s$-plane will be introduced.

The Laplace $s$-plane of the system in the fractional-order domain is given by

$$
\begin{aligned}
& s^{\alpha_{1}} X(t)=\delta(Y(t)-X(t)), \\
& s^{\alpha_{2}} Y(t)=\hat{R} X(t)-Y(t)-(\hat{R}-1) X(t) Z(t), \\
& s^{\alpha_{3}} Z(t)=(4 \gamma-b)(X(t) Y(t)-Z(t)) .
\end{aligned}
$$

Therefore, the general characteristic in the $s$-plane is given

$$
\begin{aligned}
& s^{\alpha_{1}+\alpha_{2}+\alpha_{3}}+(4 \gamma-b) s^{\alpha_{1}+\alpha_{2}} \\
& \quad+(1-b) s^{\alpha_{1}+\alpha_{3}}+\delta s^{\alpha_{2}+\alpha_{3}}+R(4 \gamma-b) s^{\alpha_{1}} \\
& \quad+\delta(4 \gamma-b) s^{\alpha_{2}}+((8 \gamma \delta-2 \delta b)((R+b)-1))=0,
\end{aligned}
$$

where the fixed points for the system are $X=Y=Z=1$ corresponding to the convection solutions. As an example, when $\alpha_{1}=0.9$ and $\alpha_{2}=\alpha_{3}=1$, we define $W=s^{\frac{1}{m}}=s^{0.1}$, then the above nonlinear equation will be a polynomial equation in $W$ as follows:

$$
\begin{aligned}
W^{29} & +\delta W^{20}+(4 \gamma-2 b+1) W^{19}+\delta(4 \gamma-b) W^{10} \\
& +R(4 \gamma-b) W^{9}+(8 \gamma \delta-2 \delta b)((R+b)-1)=0 .
\end{aligned}
$$

There are 29 roots for each value of $R$, some of them can be re-transformed back into the $s$-plane, which is called the physical plane, and the others cannot. Only the roots whose angles satisfy (19) can exist in the physical $s$-plane [23].

$$
|\angle W| \leq \frac{\pi}{m}=2 \theta .
$$

When $m=10$, the $s$-plane is represented by tenth, the $W$-plane as shown in Figures 4 , 5, 6 ( $W$-plane) by the outer dotted lines, while the inner dotted lines represent the equivalent $\pm j \omega$ axis in the $s$-plane. Thus any poles between the inner and outer dotted lines are equivalent to the left half of the $s$-plane (stable poles). All possible roots in the $W$-plane in the case when $R \in[0.1,30]$ are illustrated in Figure 4 ( $s$-plane) with step 0.001 . However, Figure 4 ( $s$-plane) shows only the poles in the physical $s$-plane through the transformation $s=W^{10}$ for poles between the outer dotted lines in Figure 4 ( $W$-plane). In addition, Figure 4 shows the pole locations versus the Rayleigh number $R$, when $R$ spans the range $[0.1,30]$. From this figure, the poles at $R=30$ are located in the right-half plane producing unstable performance. However, as $R$ decreases, the real pole goes into the negative 
direction entering the left half plane. The clear changes of the Rayleigh number $R$ from $G=0.1$ (weak heating) to $G=10$ (strong heating) affect the stability at the same points as shown in Figures 5-6. Moreover, Figures 5 and 6 show the pole locations versus the Rayleigh number $R$, when $R$ spans the range [1,26.3] and [1,20], respectively.

\section{Active control technique}

Assume that we have two different chaotic systems, one of them is the master system and the other is the slave one. We need to change the response of the slave system to synchronize with the master chaotic system via active control functions. These functions affect only the slave system without making any loading on the master chaotic response.

We define the drive master (Vadasz and Olek system [26]) and response slave systems (Jawdat et al. [29]) as follows:

$$
\begin{aligned}
& D^{\alpha_{1}} x_{1}=\delta\left(y_{1}-x_{1}\right)-s_{1} u_{x}(t), \\
& D^{\alpha_{2}} y_{1}=\hat{R}_{1} x_{1}-y_{1}-\left(\hat{R}_{1}-1\right) x_{1} z_{1}+s_{1} u_{y}(t), \\
& D^{\alpha_{3}} z_{1}=4 \gamma\left(x_{1} y_{1}-z_{1}\right)+s_{1} u_{z}(t), \\
& D^{\alpha_{1}} x_{2}=\delta\left(y_{2}-x_{2}\right)-s_{2} u_{x}(t), \\
& D^{\alpha_{2}} y_{2}=\hat{R}_{2} x_{2}-(1-b) y_{2}-\left(\hat{R}_{2}-1+b\right) x_{2} z_{2}+s_{2} u_{y}(t), \\
& D^{\alpha_{3}} z_{2}=(4 \gamma-b)\left(x_{2} y_{2}-z_{2}\right)+s_{2} u_{z}(t) .
\end{aligned}
$$

We notice that the drive system is the Vadasz and Olek [26] system, while the slave system is the Jawdat et al. [29] system, where $s_{1}$ and $s_{2}$ are on-off parameters (digital bit) which are either ' 1 ' or ' 0 ' according to the required dependence between both systems.

The unknown terms $\left(u_{x}, u_{y}, u_{z}\right)$ are active control functions to be determined,we define the error functions as follows:

$$
\begin{aligned}
& e_{x}=x_{2}-x_{1}, \\
& e_{y}=y_{2}-y_{1}, \\
& e_{z}=z_{2}-z_{1} .
\end{aligned}
$$

System (26) together with Eqs. (20)-(22) and (23)-(25) yields the error system

$$
\begin{aligned}
D^{\alpha_{1}} e_{x}= & \delta\left(e_{y}-e_{x}\right)+\left(s_{2}+s_{1}\right) u_{x}(t), \\
D^{\alpha_{2}} e_{y}= & \hat{R}_{2} e_{x}+\left(\hat{R_{2}}-\hat{R_{1}}\right) x_{1}-(1-b)\left(e_{y}+y_{1}\right) \\
& -\left(\hat{R_{2}}-\hat{R_{1}}+b\right) x_{1} z_{1}-e_{x}\left(\hat{R_{2}}-1+b\right)\left(z_{1}+e_{z}\right) \\
& -\left(\hat{R_{2}}-1+b\right) e_{z} x_{1}+y_{1}+\left(s_{2}+s_{1}\right) u_{y}(t), \\
D^{\alpha_{3}} e_{z}= & -b\left(x_{1} y_{1}-z_{1}\right)+(4 \gamma-b)\left(e_{x}\left(y_{1}+e_{y}\right)+x_{1} e_{y}-e_{z}\right) \\
& +\left(s_{2}+s_{1}\right) u_{z}(t) .
\end{aligned}
$$

We define active control functions $u_{x}(t), u_{y}(t)$ and $u_{z}(t)$ as

$$
\left(s_{2}+s_{1}\right) u_{x}(t)=V_{x}-\delta e_{y}
$$


Figure 1 The graphs represent the projection of the solution data points onto $Y-X$ plane for $\delta=5, \gamma=0.5, R=40$ and $G=5$ with different values of time delay $\tau$ and integer orders $\alpha_{1}=\alpha_{2}=\alpha_{3}=1$.

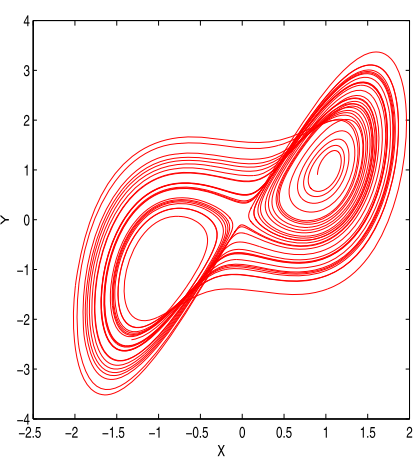

(a) $\tau=0$

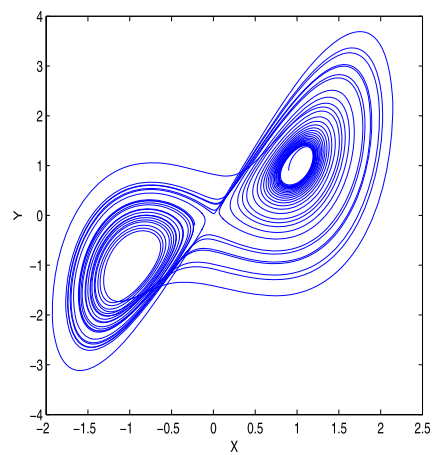

(b) $\tau=0.005$

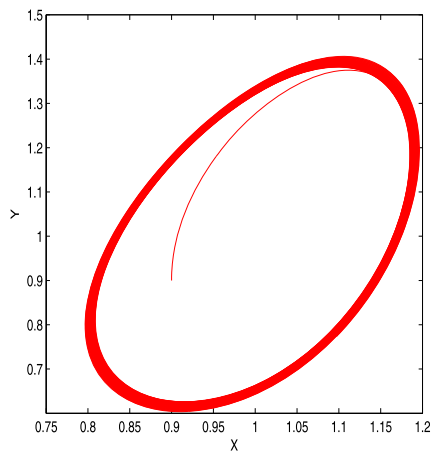

(c) $\tau=0.006$

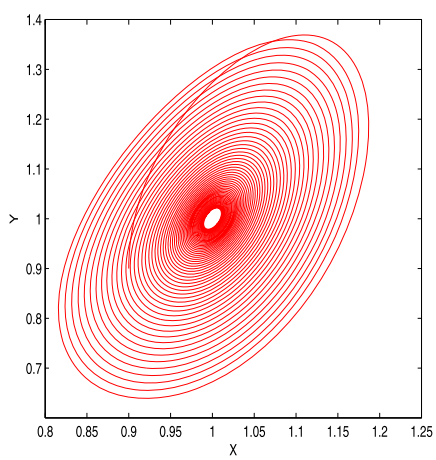

(d) $\tau=0.007$ 
Figure 2 The graphs represent the projection of the solution data points onto $Y-X$ plane for $\delta=5, \gamma=0.5, R=40$ and $G=5$ with time delay $\tau=0$ and fractional orders $\alpha_{1}=0.99$ and $\alpha_{2}=\alpha_{3}=1$.

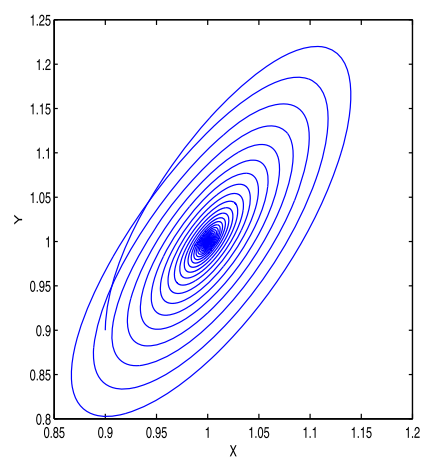

(a) $R=15$

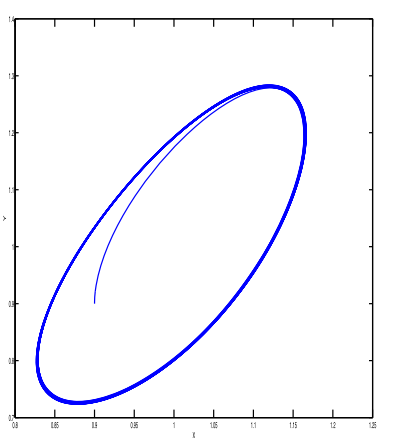

(b) $R=21.7$

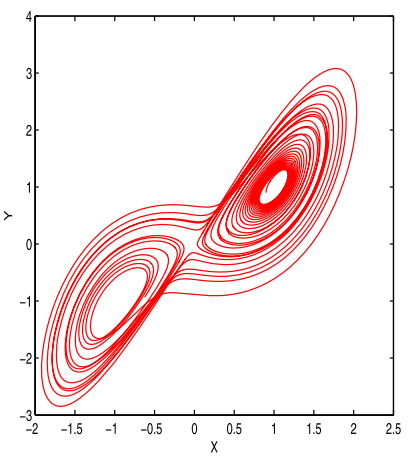

(c) $R=25$

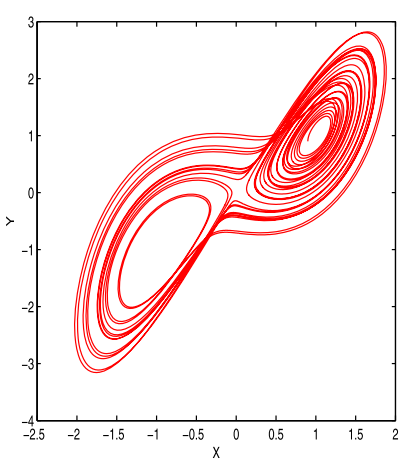

(d) $R=30$ 
Figure 3 The graphs represent the projection of the solution data points onto $Y-X$ plane for $\delta=5, \gamma=0.5, R=40$ and $G=10$ with different values of time delay $\tau$ and fractional orders $\alpha_{1}=0.99 \alpha_{2}=0.98$ and $\alpha_{3}=0.97$.

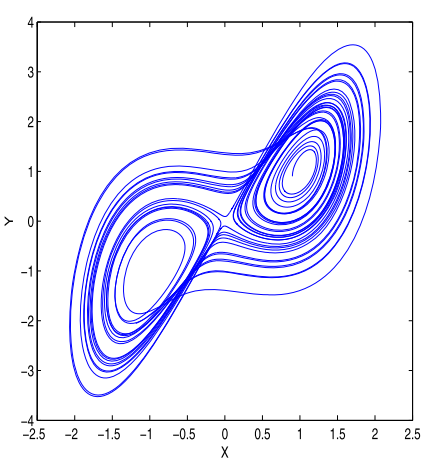

(a) $\tau=0$

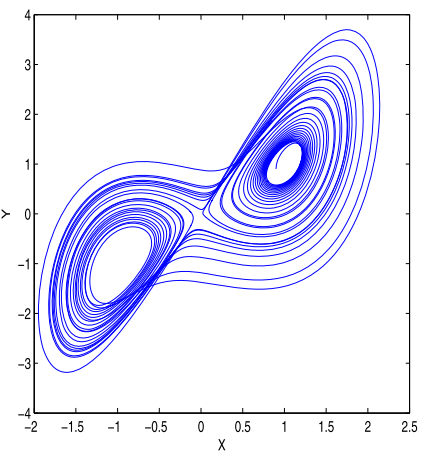

(b) $\tau=0.002$

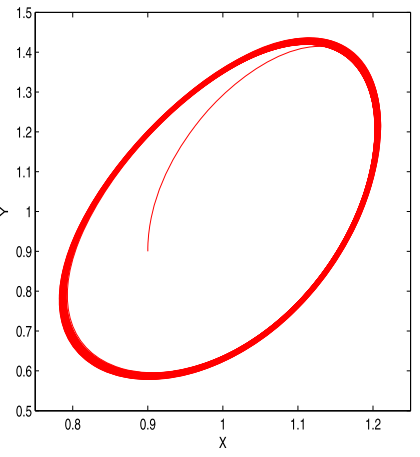

(c) $\tau=0.003$

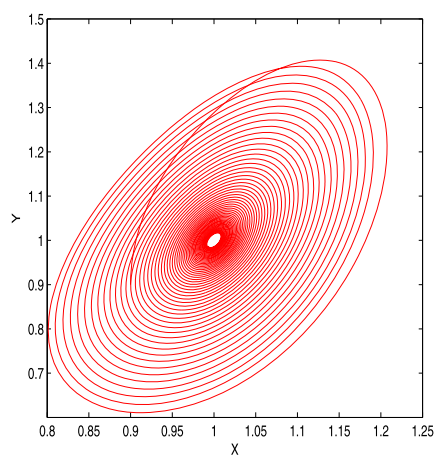

(d) $\tau=0.004$ 


$$
\begin{aligned}
\left(s_{2}+s_{1}\right) u_{y}(t)= & V_{y}-\hat{R_{2}} e_{x}-\left(\hat{R_{2}}-\hat{R_{1}}\right) x_{1}+(1-b) y_{1} \\
& +\left(\hat{R_{2}}-\hat{R}_{1}+b\right) x_{1} z_{1}+e_{x}\left(\hat{R_{2}}-1+b\right) z_{2} \\
& +\left(\hat{R_{2}}-1+b\right) e_{2} x_{1}-y_{1}, \\
\left(s_{2}+s_{1}\right) u_{z}(t)= & V_{z}-b\left(x_{1} y_{1}-z_{1}\right)-(4 \gamma-b)\left(e_{x} y_{2}+x_{1} e_{y}\right) .
\end{aligned}
$$

The terms $V_{x}, V_{y}$ and $V_{z}$ are linear functions of the error terms $e_{x}, e_{y}$ and $e_{z}$, with the choice of $u_{x}, u_{y}$ and $u_{z}$. The error system between the two chaotic systems (26) becomes

$$
\begin{aligned}
& D^{\alpha_{1}} e_{x}=-\delta e_{x}+V_{x}, \\
& D^{\alpha_{2}} e_{y}=(b-1) e_{y}+V_{y}, \\
& D^{\alpha_{3}} e_{z}=(b-4 \gamma) e_{z}+V_{z} .
\end{aligned}
$$

In fact we do not need to solve (33)-(35) if the solution converges to zero. Therefore, the control terms $V_{x}\left(e_{x}\right), V_{y}\left(e_{y}\right)$ and $V_{z}\left(e_{z}\right)$ can be chosen such that system (33)-(35) becomes stable with zero steady state.

$$
\left(\begin{array}{c}
V_{x} \\
V_{y} \\
V_{z}
\end{array}\right)=A\left(\begin{array}{c}
e_{x} \\
e_{y} \\
e_{z}
\end{array}\right)
$$

where $A$ is a $3 \times 3$ real matrix chosen so that all eigenvalues $\lambda_{i}$ of system (36) satisfy the following condition:

$$
\left|\arg \left(\lambda_{i}\right)\right|>\frac{\alpha \pi}{2}
$$

We choose

$$
A=\left(\begin{array}{ccc}
\delta-k & 0 & 0 \\
0 & (1-b)-k & 0 \\
0 & 0 & 4 \gamma-b-k
\end{array}\right) .
$$

Then the eigenvalues of the linear system (36) are equal $(-k,-k,-k)$, which is enough to satisfy the necessary and sufficient condition (37) for all fractional orders $\alpha_{1}, \alpha_{2}, \alpha_{3}<2$. In the following cases, we take $k=1$ for simplicity.

\section{Results and discussion}

In this section, we present some numerical simulations of the fractional chaotic system (4)-(6) for the time domain $0 \leq t \leq 40$. All calculations were done using Matlab with step size 0.001 , fixing the values $\delta=5, \gamma=0.5$ and taking the initial conditions $X(0)=Y(0)=$ $Z(0)=0.9$.

Figures 1-3 show how the value of $\mathrm{R}$ affects the system behavior as shown in the $X-Y$ projection with time delay, different values of $R$ and fractional derivatives, respectively. It is clear that when $R$ is small, the system exhibits steady state response; as $R$ increases, the chaotic behavior appears for wide range; and the system behaves chaotically when the fractional orders are close to 1 . For the integer case when $\alpha_{1}=\alpha_{2}=\alpha_{3}=1$, system (4)-(6) 

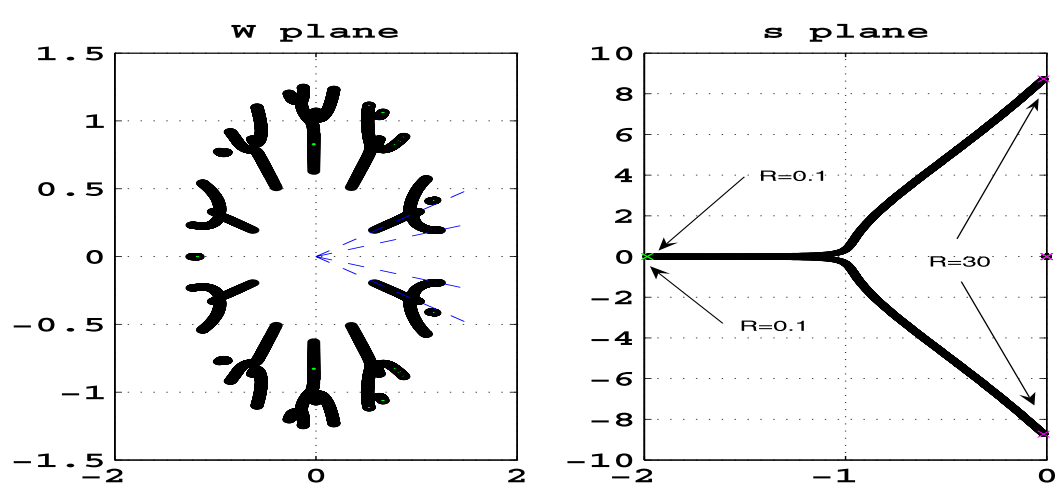

Figure 4 Poles in $W$-plane and s-plane with $G=0.1$ and fractional orders $\alpha_{1}=0.99$ and $\alpha_{2}=\alpha_{3}=1$.
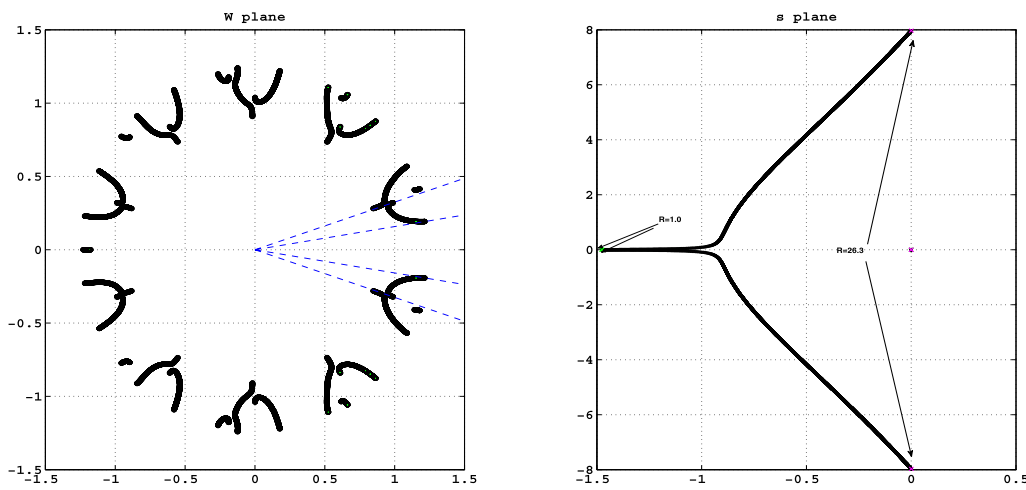

Figure 5 Poles in $W$-plane and $s$-plane $G=3$ and fractional orders $\alpha_{1}=0.99$ and $\alpha_{2}=\alpha_{3}=1$.
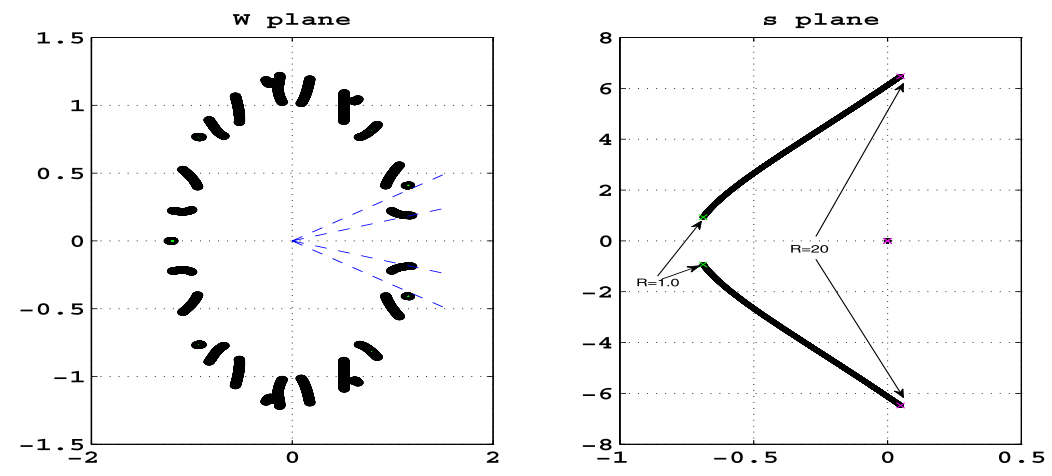

Figure 6 Poles in $W$-plane and s-plane $G=10$ and fractional orders $\alpha_{2}=\alpha_{3}=1$.

shows chaotic behavior for $0 \leq \tau \leq 0.005$, and when $\tau$ is increased further, the system tends to periodicity ( $\tau=0.006$ ) and becomes stable when $\tau=0.007$. Figure 2 shows the effect of the fractional order $\alpha_{1}=0.99, \alpha_{2}=\alpha_{3}=1$ for system (4)-(6) with different values of Rayleigh number $R$ and without time delay $(\tau=0)$. It is clear that the chaotic solution takes over and the convection loses its stability when $R$ increases from 15 to 30 . For the 

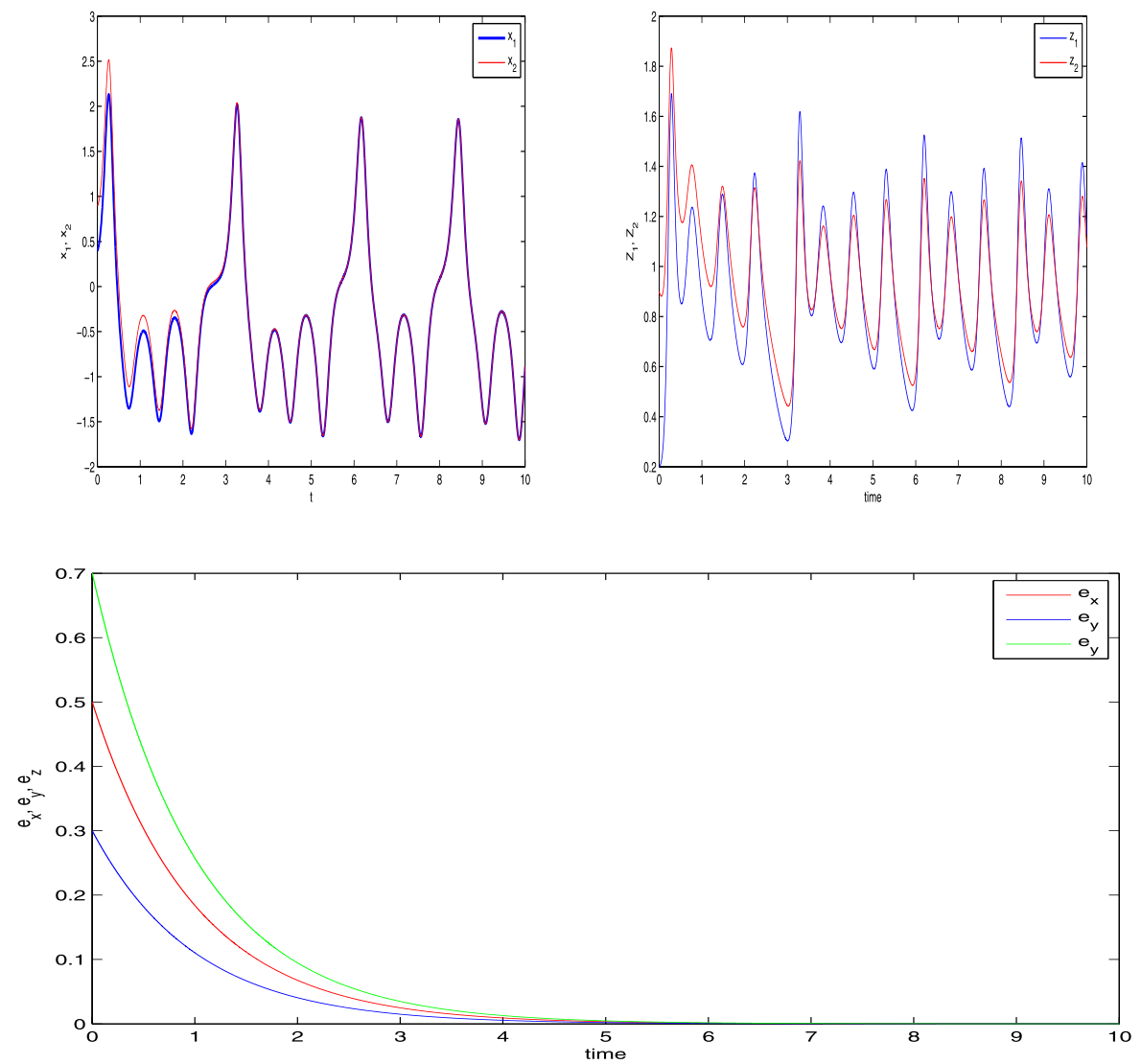

Figure 7 Time domain response for $x_{1}, x_{2}, z_{1}, z_{2}$ and the error functions $e_{x}, e_{y}$ and $e_{z}$ for both systems when $\alpha_{1}=\alpha_{2}=\alpha_{3}=1$ and $\delta=5, \gamma=0.5, R=40$.

case of fractional order $\left(\alpha_{1}=0.99, \alpha_{2}=0.98, \alpha_{3}=0.97\right)$ and with time delay $0 \leq \tau \leq 0.004$, the system gets stabilized for smaller values of $\tau(\tau=0.004)$, as shown in Figure 3.

Figures 7 and 8 show the Vadasz and Olek system [26] and the Jawdat et al. [29] system responses, the error function and the active control signals versus the time when $\alpha_{1}=\alpha_{2}=$ $\alpha_{3}=1$ and $\alpha_{1}=\alpha_{2}=\alpha_{3}=0.99$, respectively. We see that when $\left(s_{1}, s_{2}\right)=(0,1)$ the Vadasz and Olek system [26] works normally and the Jawdat et al. [29] system adapts its response to follow the Vadasz and Olek system [26]. Therefore the first system works normally without any loading effect, and the second system adapts its response to synchronize with the first system with initial conditions $X(0)=0.4, Y(0)=0.6, Z(0)=0.2$. and $X(0)=Y(0)=$ $Z(0)=0.9$. Similarly, the Jawdat et al. [29] system works individually, and the Vadasz and Olek system [26] follows exactly the Jawdat et al. [26] system when $\left(s_{1}, s_{2}\right)=(1,0)$, and the two systems work independently (no synchronization) when $\left(s_{1}, s_{2}\right)=(0,0)$.

\section{Conclusions}

In this paper, the effect of time delay with fractional order in a fluid saturated porous layer subjected to gravity and heated from below under the effect of various values of the Rayleigh number $R$ has been studied. Several cases of fractional derivatives are introduced with stability analysis. Static and dynamic synchronization has been obtained using the active control by changing the switching parameters. Numerical simulations allowed us 

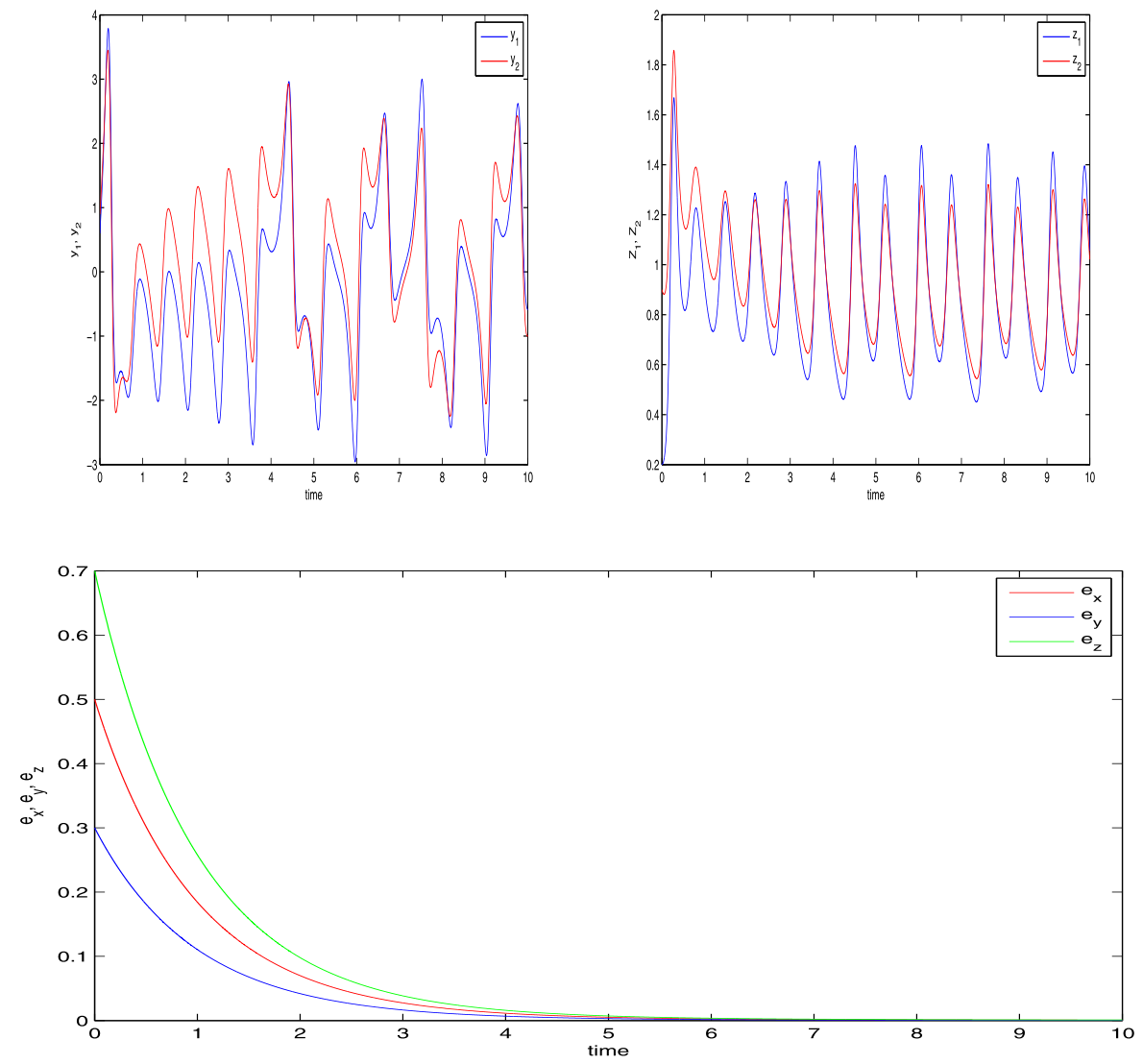

Figure 8 Time domain response for $y_{1}, y_{2}, z_{1}, z_{2}$ and the error functions $e_{x}, e_{y}$ and $e_{z}$ for both systems when $\alpha_{1}=\alpha_{2}=\alpha_{3}=1$ and $\delta=5, \gamma=0.5, R=40$.

to observe that the clear changes of that chaotic behavior get stabilized for some values of time delay, Rayleigh number and the fractional order changes.

Acknowledgements

The author expresses his thanks to unknown referees for the careful reading and helpful comments.

\section{Competing interests}

The author declares that they have no competing interests.

\section{Author's contributions}

All authors read and approved the final manuscript.

\section{Publisher's Note}

Springer Nature remains neutral with regard to jurisdictional claims in published maps and institutional affiliations.

Received: 5 January 2017 Accepted: 21 September 2017 Published online: 04 October 2017

\section{References}

1. Moaddy, K, Radwan, A, Salama, K, Momani, S, Hashim, I: The fractional-order modeling and synchronization of electricall coupled neuron systems. Comput. Math. Appl. 64(10), 3329-3339 (2012)

2. Feki, M: An adaptive chaos synchronization cheme applied to secure communication. Chaos Solitons Fractals 18(1), $141-148$ (2003)

3. Radwan, A, Soliman, A, El-Sedeek, A: An inductorless cmos realization of Chua's circuit. Chaos Solitons Fractals 18 , 149-158 (2003)

4. Radwan, A, Soliman, A, El-Sedeek, A: Mos realization of the modified Lorenz chaotic system. Chaos Solitons Fractals 21, 553-561 (2004) 
5. Radwan, A, Moaddy, K, Momani, S: Stability and nonstandard finite difference method of the generalized Chua's circuit. Comput. Math. Appl. 62, 961-970 (2011)

6. Pecora, L, Carrol, T: Synchronization in chaotic systems. Phys. Rev. Lett. 64, $821-824$ (1990)

7. Pecora, L, Carrol, T: Driving systems with chatic signals. Phys. Rev. A 44(4), 2374-2383 (1991)

8. Pinto, CMA: Strange dynamics in a fractional derivative of complex-order network of chaotic oscillators. Int. J. Bifurc. Chaos 25(1), 1550003 (2015)

9. Yan, J, Li, C: On chaos synchronization of fractional differential equations. Chaos Solitons Fractals 32(2), 725-735 (2007)

10. Chua, L, Makoto, I, Kocarev, L, Eckert, K: Chaos synchronization in chuas circuit. J. Circuits Syst. Comput. 3(1), 93-108 (1993)

11. Pinto, CMA, Carvalho, ARM: Strange patterns in one ring of Chen oscillators coupled to a buffer cell. J. Vib. Control 22, 3267-3295 (2014)

12. Sun, K, Sprott, J: Bifurcations of fractional-order diffusionless Lorenz system. Electron. J. Theor. Phys. 6(22), 123-134 (2009)

13. Podlubny, I: Fractional Differential Equations. Academic Press, New York (1999)

14. Luo, C, Wang, X: Chaos generated from the fractional-order complex Chen system and its application to digital secure communication. Int. J. Mod. Phys. C 24, 1350025 (2013)

15. Muthukumar, P, Balasubramaniam, P, Ratnavelu, K: Fast projective synchronization of fractional order chaotic and reverse chaotic systems with its application to an affine cipher using date of birth (dob). Nonlinear Dyn. 80 1883-1897 (2015)

16. Zhang, F: Lag synchronization of complex Lorenz system with applications to communication. Entropy 17, 4974-4985 (2015)

17. $\mathrm{Xu}, \mathrm{F}, \mathrm{Cressman}, \mathrm{R}, \mathrm{Shu}, \mathrm{X}-\mathrm{B}, \mathrm{Liu}, \mathrm{X}$ : A series of new chaotic attractors via switched linear integer order and fractional order differential equations. Int. J. Bifurc. Chaos 25(1), 1550008 (2015)

18. $\mathrm{Xu}, \mathrm{F}$ : Integer and fractional order multiwing chaotic attractors via the Chen system and the Lü system with switching control. Int. J. Bifurc. Chaos 24, 1450029 (2014)

19. $\mathrm{Xu}, \mathrm{F}, \mathrm{Pei}, \mathrm{Y}, \mathrm{Xiaoxin,} \mathrm{L}$ : Synchronization and stabilization of multi-scroll integer and fractional order chaotic attractors generated using trigonometric functions. Int. J. Bifurc. Chaos 23, 1350145 (2013)

20. Yu, Y, Li, H, Su, Y: The synchronization of three chaotic fractional-order Lorenz systems with bidirectional coupling. Int. J. Bifurc. Chaos 20(1), 1-15 (2010)

21. Odibat, ZM, Corson, N, Aziz-Alaoui, MA, Bertellec, C: Synchronization of chaotic fractional-order systems via linear control. Int. J. Bifurc. Chaos 20, 81 (2010)

22. Bhalekar, S, Daftardar-Gejji, V: Antisynchronization of nonidentical fractional-order chaotic systems using active control. Int. J. Differ. Equ. 2011250763 (2011)

23. Zhen, W, Xia, H, Ning, L, Xiao-Na, S: Image encryption based on a delayed fractional-order chaotic logistic system. Chin. Phys. B 21(5), 050506 (2012)

24. Daftardar-Gejji, V, Bhalekar, S, Gade, P: Dynamics of fractional-ordered Chen system with delay. Pramana 79, 61-69 (2012)

25. Sheu, L, Tam, L, Chen, J, Chen, H, Lin, K, Kang, Y: Chaotic convection of viscoelastic fluids in porous media. Chaos Solitons Fractals 37, 113-124 (2008)

26. Vadasz, P, Olek, S: Weak turbulence and chaos for low Prandtl number gravity driven convection in porous media. Transp. Porous Media 37, 69-91 (1999)

27. Vadasz, P, Olek, S: Transition and chaos for free convection in a rotating porous layer. Int. J. Heat Mass Transf. 41, 1417-1435 (1974)

28. Moaddy, K, Radwan, A, Hashim, I, Jawdat, J: Bifurcation behaviourand control on chaotic convection of nanofluids with fractional-orders. In: Fujita, H, Tuba, M (eds.) Recent Advances in Mathematical Methods and Computational Techniques in Modern Science, 23-25 April 2013; Japan, pp. 63-73 (2013). WESEAS

29. Jawdat, J, Hashim, I: Low Prandtl number chaotic convection in porous media with uniform internal heat generation. Int. Commun. Heat Mass Transf. 37, 629-636 (2010)

30. Lorenz, E: Deterministic non-periodic flow. J. Atmos. Sci. 20, 130-142 (1963)

31. Sparrow, C: The Lorenz Equations: Bifurcations, Chaos, and Strange Attractors. Springer, New York (1982)

32. Diethelm, K, Ford, N: Analysis of fractional differential equations. J. Math. Anal. Appl. 265, 229-248 (2002)

33. Diethelm, K, Ford, N, Freed, A: Detailed error analysis for a fractional Adams method. Numer. Algorithms 26, 31-52 (2006)

\section{Submit your manuscript to a SpringerOpen ${ }^{\circ}$ journal and benefit from:}

- Convenient online submission

- Rigorous peer review

- Open access: articles freely available online

- High visibility within the field

- Retaining the copyright to your article

Submit your next manuscript at $\gg$ springeropen.com 